\title{
Nonlinear waves and instabilities leading to secondary reconnection in reconnection outflows
}

\author{
Giovanni Lapenta ${ }^{1,2, \dagger}$, Francesco Pucci ${ }^{1}$, Vyacheslav Olshevsky ${ }^{1}$, \\ Sergio Servidio ${ }^{3}$, Luca Sorriso-Valvo ${ }^{4}$, David L. Newman ${ }^{5}$ and \\ Martin V. Goldman ${ }^{5}$ \\ ${ }^{1}$ Center for Mathematical Plasma Astrophysics, Department of Mathematics, KULeuven Belgium, 200B \\ Celestijnenlaan, Leuven, B-3001 \\ ${ }^{2}$ Space Science Institute, Boulder, USA \\ ${ }^{3}$ Via P. Bucci, Cubo 31C, Arcavacata di Rende, I-87036, Dipartimento di Fisica, \\ Università della Calabria \\ ${ }^{4}$ Nanotec-CNR, U.O.S. Cosenza, Arcavacata di Rende, Italy \\ ${ }^{5}$ University of Colorado, Boulder, CO 80309, USA
}

(Received 10 August 2017; revised 3 January 2018; accepted 3 January 2018)

Reconnection outflows have been under intense recent scrutiny, from in situ observations and from simulations. These regions are host to a variety of instabilities and intense energy exchanges, often even superior to the main reconnection site. We report here a number of results drawn from an investigation of simulations. First, the outflows are observed to become unstable to drift instabilities. Second, these instabilities lead to the formation of secondary reconnection sites. Third, the secondary processes are responsible for large energy exchanges and particle energization. Finally, the particle distribution function are modified to become non-Maxwellian and include multiple interpenetrating populations.

Key words: astrophysical plasmas, plasma simulation, space plasma physics

\section{Introduction}

The research of the last two decades has shown that kinetic reconnection is a fast process that develops on Alfvén time scales (Biskamp 2000). This result is a spectacular success for kinetic modelling (Birn et al. 2001), now confirmed in situ by the Magnetospheric Multiscale Mission (Burch et al. 2016). However, fast kinetic reconnection is not the solution to all of the problems in reconnection: fast kinetic reconnection has thus far been observed and modelled only in localized regions. Instead, in many astrophysical and laboratory systems, large amounts of energy are converted over large domains. How can we bring fast kinetic reconnection to large scales?

A possible scenario to reach large energy conversion rates at system scales is to imagine a situation where the initiation of reconnection is followed by a chain reaction

$\dagger$ Email address for correspondence: giovanni.lapenta@kuleuven.be 
of more and more secondary reconnection sites (Bulanov, Sakai \& Syrovatskii 1979; Loureiro, Schekochihin \& Cowley 2007; Lapenta 2008; Tenerani et al. 2016). Under these conditions, reconnection tends to become chaotic with many reconnection sites being spawned by instability and reabsorbed by island coalescence, leading to fast reconnection (Bhattacharjee et al. 2009; Skender \& Lapenta 2010; Pucci \& Velli 2013; Huang, Comisso \& Bhattacharjee 2017).

Three-dimensional reconnection is accompanied by many more instabilities than just the formation of secondary islands in the primary reconnection site seen in two-dimensional reconnection: the reconnection inflow (Daughton et al. 2011) and the reconnection outflow (Lapenta et al. 2015) host instabilities that lead to secondary reconnection. The first mechanism is primarily present in reconnection separatrices in the case of strong guide fields (Lapenta, Wang \& Cazzola 2016c), while the latter is present at all guide fields (Lapenta et al. 2014b).

Outflows from reconnection are rich in free energy that can drive instabilities. Among the possibilities we consider here:

(i) Velocity shears around the outflow jet that can drive Kelvin-Helmholtz instability (Lottermoser, Scholer \& Matthews 1998).

(ii) Density and temperature gradients at the front formed by the outflowing jet interacting with the ambient plasma leads to drift-type instabilities (Divin et al. 2015b).

(iii) Unfavourable curvature of field lines between the separatrices in the outflow region can lead to interchange (Rayleigh-Taylor-type) instabilities (Nakamura, Matsumoto \& Fujimoto 2002; Guzdar et al. 2010; Lapenta \& Bettarini 2011).

(iv) Flux ropes in the outflows may be kink unstable (Shafranov 1957; Kruskal \& Tuck 1958).

(v) Additional instabilities are caused by phase-space features such as anisotropies leading to whistler waves and beams leading to streaming instabilities (Goldman, Newman \& Lapenta 2016).

All these instabilities can cause strong deformation of the flow, leading possibly to turbulence (Pucci et al. 2017), energy exchange (Lapenta et al. 2016b) and secondary reconnection (Lapenta et al. 2015).

The three-dimensional scenario for large-scale turbulence is one where reconnection might lead to a chain reaction type of sequence of events. Reconnection is initiated at one location but the instabilities associated with the flows and the other sources of free energy induced by reconnection lead to the formation of secondary reconnection sites. While not yet observed in simulation, this scenario on large scales (not yet accessible to simulation) can then progress in successive generations of tertiary and further reconnection sites, filling macroscopic domains.

Below, we organize our material as follows. Section 2 reports the type of simulations we use to analyse the reconnection outflows and the instabilities developing there. Section 3 investigates the fluctuation spectrum produced in the outflow. Section 4 discusses how the fluctuations interact with the particles energizing them. Conclusions and future directions are outlined in $\S 5$.

\section{Development of outflow instabilities and secondary reconnection}

In order to study the properties of outflow instabilities and secondary reconnection, we use particle-in-cell numerical simulations. 
We consider here the same run previously considered in Lapenta et al. (2015). The system is initialized with a Harris equilibrium (Harris 1962)

$$
\boldsymbol{B}=B_{0 x} \tanh (y / \delta) \boldsymbol{e}_{x}+B_{0 z} \boldsymbol{e}_{z}, \quad n=n_{0 b}+\frac{n_{0}}{\cosh ^{2}(y / \delta)} .
$$

uniquely specified by the mass ratio $m_{i} / m_{e}=256$, the temperature ratio $T_{i} / T_{e}=5$ and $v_{t h, e} / c=0.045$. We set the density of the uniform plasma background to $n_{0 b}=$ $n_{0} / 10$, the value of the guide field to $B_{0 z} / B_{0 x}=1 / 10$ and the thickness of the initial current sheet to $\delta=0.5 d_{i}$. The evolution is followed using the fully electromagnetic and fully kinetic iPic3D code (Markidis, Lapenta \& Rizwan-uddin 2010) that treats both electrons and ions as particles. Details are provided in Lapenta et al. (2015). We use coordinates where $x$ is along the initial magnetic field, $y$ is along the initial gradients and $z$ is along the initial current. Open boundary conditions are imposed in the $x$ and $y$ direction and periodicity is imposed along $z$. We consider a threedimensional box of shape $[40.0,15.0,10.0] d_{i}$, where $d_{i}$ is the ion inertial length, which is resolved by a Cartesian grid of $[512,192,128]$ cells, each one populated with 125 particles. The spatial resolution is $\Delta x=1.25 d_{e}$, where $d_{e}$ is the electron inertial length, and the time step is $\Delta t=\pi / 10 \omega_{c e}^{-1}$, where $\omega_{c e}$ is the electron gyro-frequency.

Reconnection is initialized in the centre with an initial $x$-shaped perturbation that leads to the formation of a central $X$-line. A reconnection site develops with plasma accelerated towards the reconnection region and expelled out of it. The electron flow pattern in the fully developed nonlinear stage is shown in figure 1 . The electrons are first attracted toward the central $X$-line where the $z$-directed reconnection electric field accelerates them to high speed. The Lorentz force then deflects the particles towards the outflow. In this region, the system presents a remarkable invariance along $z$, resembling the same physics of two-dimensional fast kinetic reconnection.

In the outflow, however, the electron flow pattern becomes distorted and meanders about, eventually passing downstream away of the reconnection region. In this region the electron flow becomes more turbulent.

The region of electron meandering corresponds to the front formed by the interaction of the outflowing plasma with the surrounding media. At the front, an effect similar to that of a snowplough pushes the plasma outward. A form forms where at least three of the mechanisms mentioned above are present: the field lines wrap around the front gaining unfavourble curvature that can lead to interchange-type instabilities, the density gradient is unstable to drift modes and the distribution function becomes severely non-Maxwellian leading to microinstabilities.

Figure 2 shows the state of the front after the instability starts to develop. The density $(a)$ becomes rippled by a mode that presents a strong perturbation of the $E_{z}$ (b). When the mode structure of these fluctuations is Fourier analysed, the resulting spectrum in $k_{z}$ is reported in $(c)$. The observed features are characteristic of a drift mode in the lower hybrid range.

The identification of the instability as having primarily the nature of a lower hybrid drift instability (LHDI) is confirmed by the temporal spectrum measured at a fixed point reached by the front (Divin et al. 2015a,b). A spectrogram, obtained with standard windowing methods similar to those used on board real space probes, is reported in figure 3: the observed frequency spectrum is reported at different times. Panel $(b)$ reports the corresponding observed local magnetic field intensity. When the front arrives, an intense signal in the lower hybrid range is measured.

As the evolution is continued, the ripples in the front become more intense and start to interact leading to conditions where magnetic field of opposite polarity is 


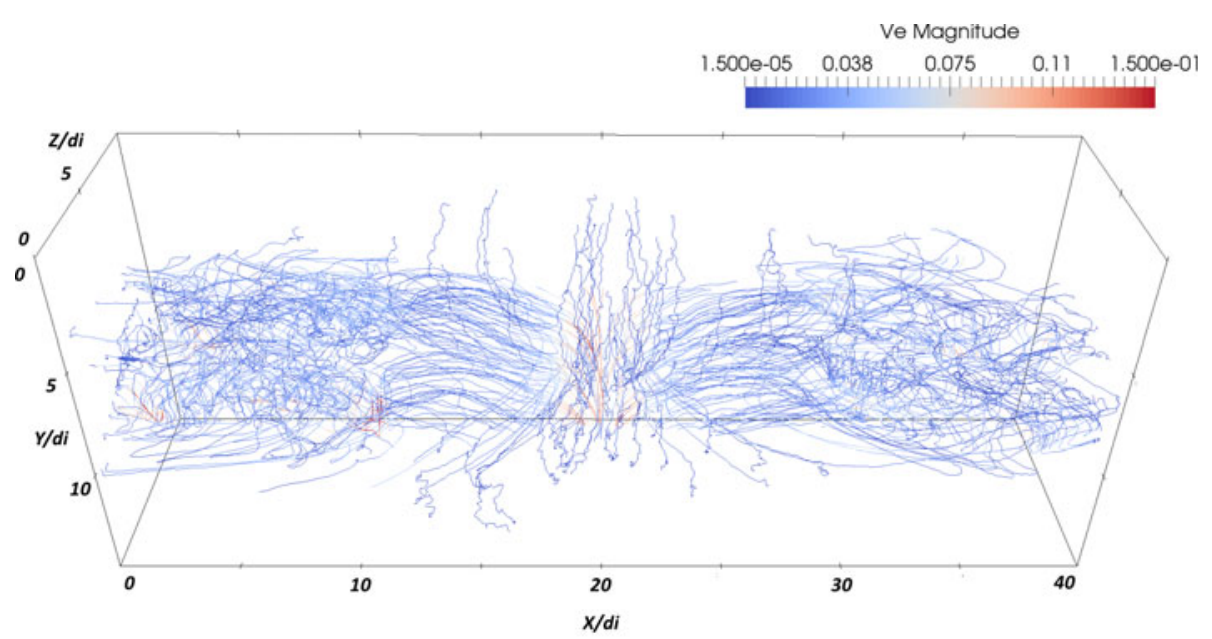

FIGURE 1. Visualization of the electron flow around a reconnection site. We report streamlines of the first-order moment of the electron distribution (the electron flow velocity) coloured by the intensity of the local electron speed (normalized to the speed of light).
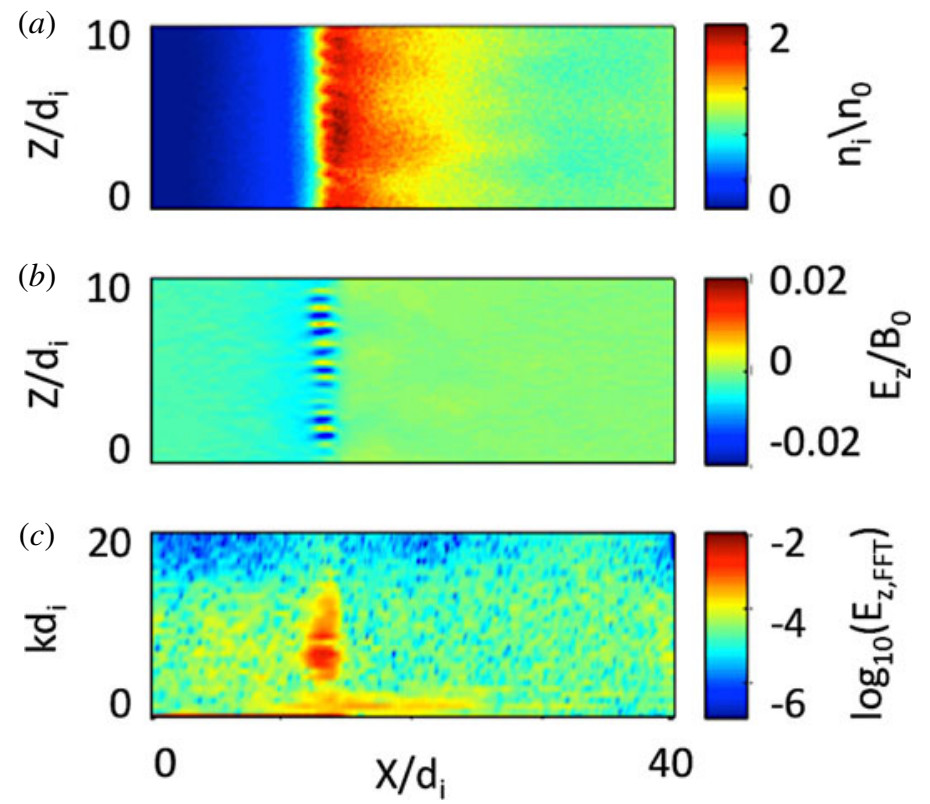

FIGURE 2. Early stages of the instability at the front, at time $\omega_{c i}^{-1}=15$. The panels show from top to bottom: ion density $(a)$, $z$-component of the electric field $(b)$ and the Fourier spectrum in $k_{z}$ of the perturbation of the electric field $E_{z}(c)$.

brought in contact promoting secondary reconnection. Figure 4 shows the front at two consecutive times: at later times, the 'fingers' formed in the front tend to interact and coalesce (Vapirev et al. 2013). 

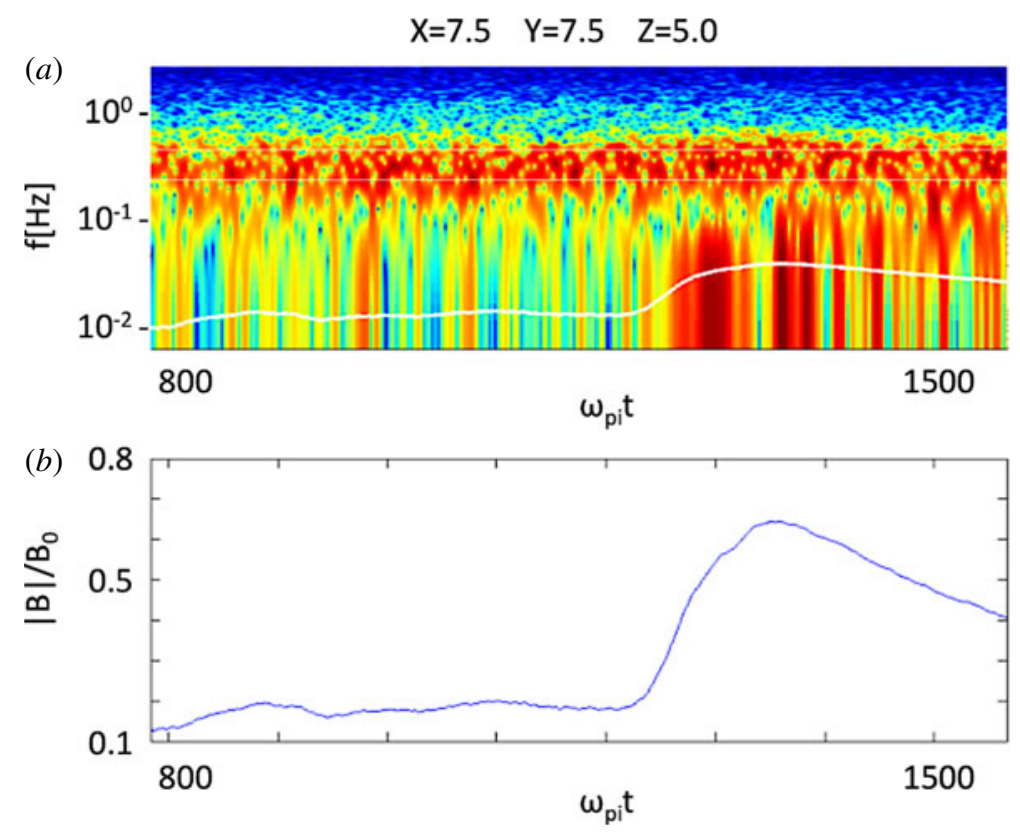

FIGURE 3. Signal from a virtual probe embedded in the simulation at $x / d_{i}=7.54, y / d_{i}=$ 7.54, $z / d_{i}=5.04$. (a) Shows the spectrogram of the $E_{z}$ signal measured. To guide the eye the local lower hybrid frequency is indicated by a white line. $(b)$ Shows the magnetic field intensity measured by the virtual probes at the different times.

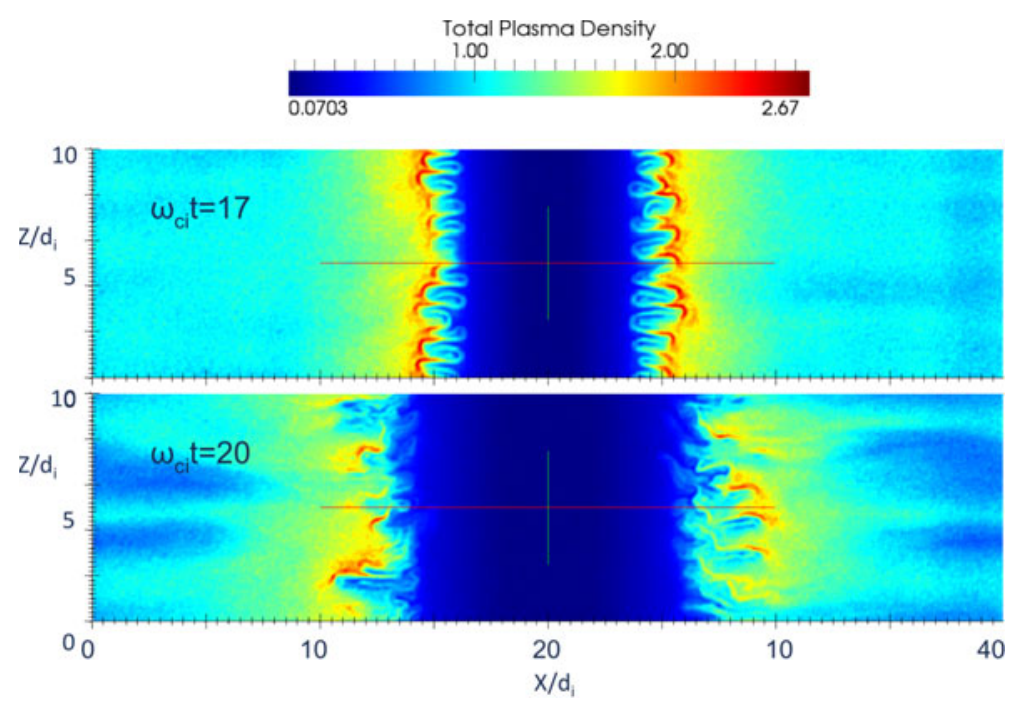

FIGURE 4. Density at the front for two different times.

Lapenta et al. (2015) analysed several indicators to detect positively secondary reconnection sites: direct analysis of field line connectivity, energy conversion in the electron frame $\left(\boldsymbol{J} \cdot\left(\boldsymbol{E}+\boldsymbol{v}_{e} \times \boldsymbol{B}\right)\right)$, electron agyrotropy, slippage $\left(\boldsymbol{v}_{e \perp}-\boldsymbol{E} \times \boldsymbol{B} / B^{2}\right)$, topological measure of field line breakage (Hesse \& Schindler 1988; Biskamp 2000) 


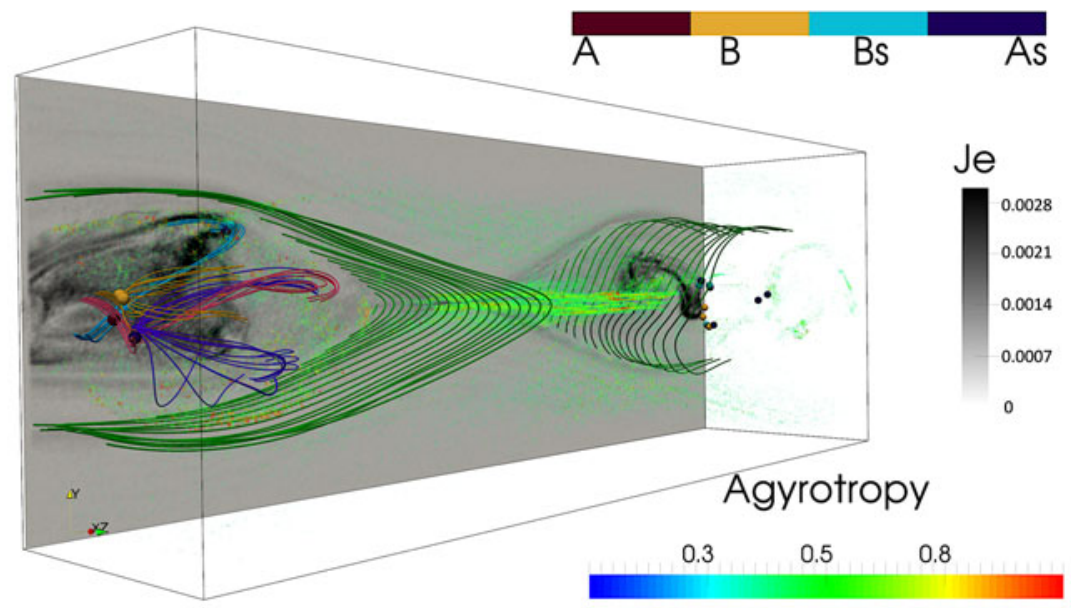

FIgURE 5. Combination of different measures at the same time: a vertical cut of the electron current intensity (grey scale); false colour volume rendering of agyrotropy; magnetic null points (coloured spheres) coloured according to their topological type. Selected field lines reconnected once at the primary site are shown in green, while secondary reconnected lines near the nulls are shown in purple, pink, orange and light blue.

$\left(\boldsymbol{b} \times \nabla \times\left(E_{\|} \boldsymbol{b}\right)\right)$, where $\boldsymbol{b}$ is the unit vector along $\boldsymbol{B}$ and $E_{\|}$is the parallel component of the non-ideal part of Ohm's law (Biskamp 2000), normalized as $e E_{\|} / m_{i} c \omega_{p i}$.

A specific orientation of the magnetic field which allows for the field annihilation and energy release, is an important indicator of magnetic reconnection. In the classical two-dimensional picture, magnetic field lines of opposite direction approach each other and form an $X$-point, which, extended to three dimensions, becomes an $X$-line denoted by the strong $Z$-aligned current in our simulation. This sort of magnetic reconnection, however, does not require the field to become exactly zero (hence, no magnetic nulls are formed) on the reconnection site. We use the technique based on the topological degree method (Greene 1992) as described in Olshevsky et al. (2016) to locate and classify magnetic nulls. Indeed, in the simulation reported here, no magnetic nulls are present in the central current sheet as summarized in figure 5. However, the diffusion region around the $X$-line is characterized by strong electron agyrotropy $A=\left(P_{e, \perp 1}-P_{e, \perp 2}\right) / P_{e, \perp 1}-\left(P_{e, \perp 2}\right)$ that is shown with volume rendering. No strong energy conversion is associated with the 'main' reconnection $X$-line.

In contrast, in the reconnection outflow a number of magnetic nulls form, which are depicted by coloured spheres in figure 5. The colour denotes the magnetic nulls' topological type: A and B (red and orange) are the three-dimensional extensions of the $X$-points called radial nulls; while As and Bs (light blue and blue) represent magnetic islands or magnetic flux ropes. Both radial and spiral nulls are present in the outflows, however the number of spiral ones is larger. Magnetic field lines in the vicinity of the null points in the left outflow are shown with the corresponding colours. A pair of spiral nulls is formed in a swirl of the light blue magnetic field lines, probably driven by a shear instability. This null pair is embedded in the region of strong energy conversion (see figure 5). Other nulls in this outflow are on the interfaces of magnetic fields of different polarities characterized by complex field patterns resembling an $X$ pattern (orange) and merging into flux ropes (purple and 
pink). Recent observations (Fu et al. 2017) provide a strong evidence that intermittent energy conversion in the reconnection outflows is associated with the spiral magnetic nulls and twisted magnetic fields.

The picture provides an indication of the scenario described in the introduction: the initial reconnection site located at the centre of the box and forming an $X$-line produces two outflows that become unstable and produce in turn secondary reconnection sites. In the process the plasma becomes effectively turbulent and a large fraction of the energy is converted to particle heat at these unstable fronts, rather than at the central $X$-line.

\section{Development of intermittent turbulence in reconnection outflows}

Above we have observed how reconnection tends to become visually turbulent. But is turbulence real? In a recent paper (Pucci et al. 2017), the properties of electric and magnetic fluctuations that are produced by magnetic reconnection have been analysed. Because of the inhomogeneous background it is important to first establish the anisotropy level and in general the three-dimensional (3-D) properties of turbulence. Analysis of the autocorrelation function of the magnetic field fluctuations have shown that the turbulence that develops in the reconnection jets is anisotropic. In particular, magnetic vortexes are elongated in the direction of the background magnetic field, namely $x$, with a second smaller anisotropy in the $(y, z)$ plane. The second anisotropy becomes negligible for smaller scales and isotropy is recovered in the $\left(k_{y}, k_{z}\right)$ plane

for $k_{y z}>1.5$, with $k_{y z}=\sqrt{k_{y}^{2}+k_{z}^{2}}$. This allows us to reduce the 3-D spatial spectra to 1-D isotropic spectra computed in the $\left(k_{y}, k_{z}\right)$ plane and integrated in $k_{x}$. The results of this computation shows magnetic and electric spectra with a clear power law in the sub-ion range $1.5<k_{y z} d_{i}<15$. As observed in space plasmas (Eastwood et al. 2009) the magnetic and electric spectra depart from each other at around $k d_{i} \sim 1$, the electric one proceeding with a spectral slope of $\sim 1$ and the magnetic one with a slope of $-8 / 3$. Recently Matteini et al. (2017), following simple dimensional arguments, have interpreted this phenomenon as due to the dominance of the Hall effect at small scales. It is worth remarking how this interpretation still holds in such an anisotropic and inhomogeneous system, where spectra need to be carefully extracted removing large-scale background profiles and border effects.

Turbulence is responsible for the transfer of energy from fields to particles. In this work we show that this energy exchange does not take place homogeneously in the reconnection events but is located in small regions in the reconnection outflows where the energy transfer is very intense. In order to quantify the energy exchange we introduce the two dissipation proxies $D_{l}=\boldsymbol{J} \cdot \boldsymbol{E}$ and $D_{i}=\boldsymbol{J} \cdot\left(\boldsymbol{E}+\boldsymbol{v}_{i} \times \boldsymbol{B}\right)$ (Zenitani et al. 2011), where $\boldsymbol{J}$ is the total current, $\boldsymbol{E}$ is the electric field, $\boldsymbol{v}_{i}$ is the ion fluid velocity and $\boldsymbol{B}$ is the magnetic field. In figure $7(a)$ the probability density functions (PDFs) of $\delta D_{l}=D_{l}-\left\langle D_{l}\right\rangle_{x, y, z}$ and $\delta D_{i}=D_{i}-\left\langle D_{i}\right\rangle_{x, y, z}$ are plotted, where \langle\rangle$_{x, y, z}$ means average along the three axes. The two PDFs are compared with the normalized Gaussian distribution (plotted with the dashed-red line). They strongly depart from Gaussian distributions, presenting instead high tails up to several standard deviations $\sigma$. In $(b)$, the average $D_{i}$ conditioned to a threshold current density is shown. The plot is constructed as follows: a threshold in the current density magnitude is considered and the average of $D_{i}$ is computed using all those points in the domain where the value of the current is bigger than the fixed threshold. This average is then normalized to the average of $D_{i}$ on all points, which gives by definition $\left\langle D_{i} \mid J=0\right\rangle /\left\langle D_{i}\right\rangle=1$. The black points in the plots represent the result of such a computation for different 

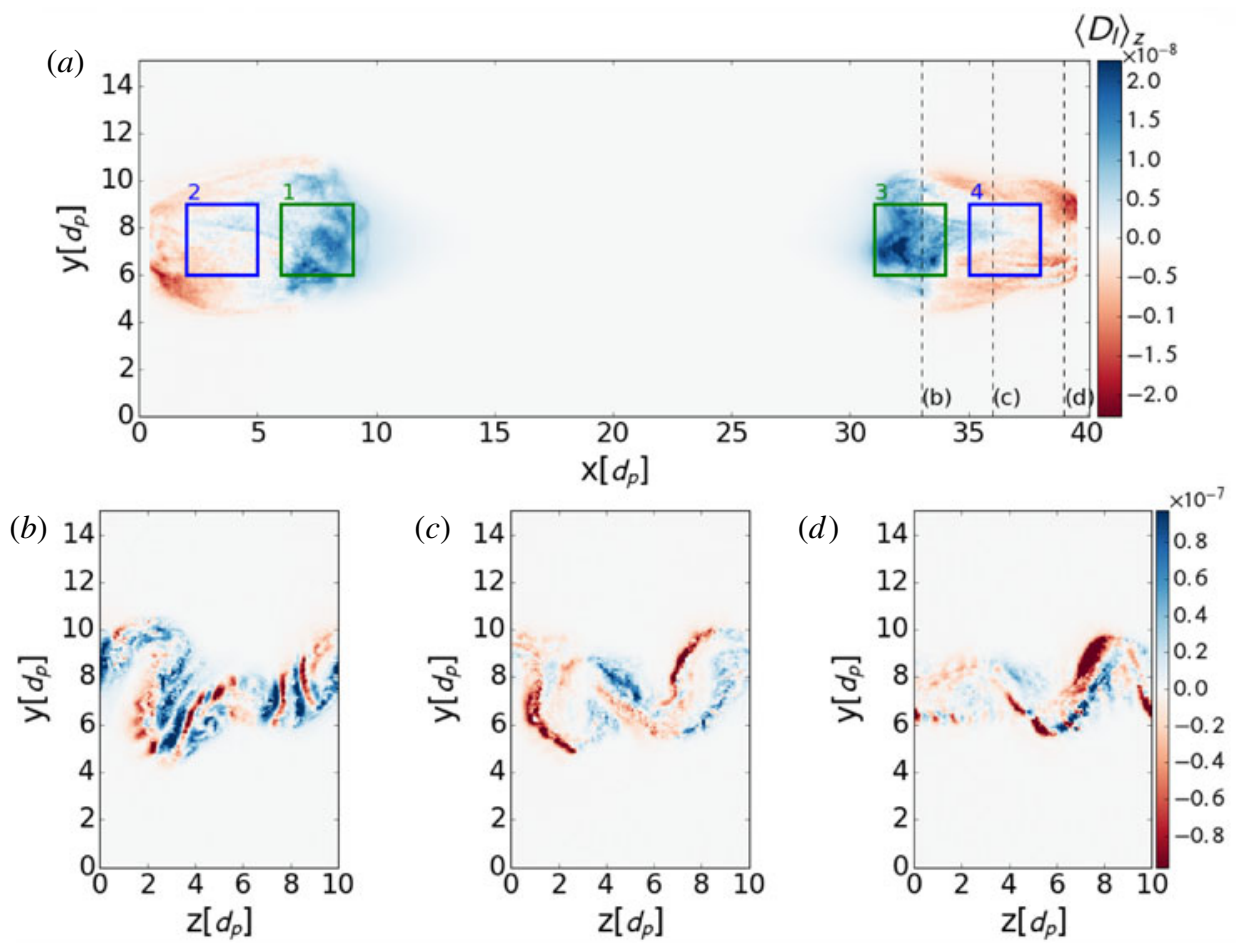

FIgURE 6. Energy exchange $D_{l}=\boldsymbol{J} \cdot \boldsymbol{E}$ in the $(x, y)$ plane averaged in the $z$ direction $(a)$, and in the $(y, z)$ plane at $x=33 d_{i}(b), x=36 d_{i}(c), x=39 d_{i}(d)$. The $X$-line is located at $x=20 d_{i}$. The three boxes in $(a)$ are the ones used for the statistical analysis presented in figure $8-9$.

values of the threshold. The blue curve represents the filling factors, i.e. the fraction of points used for computing the average with respect to the total number of points in the sample. The average of $D_{i}$ strongly increases when higher threshold are considered up to $J / J_{r m s}=10$, where $J_{r m s}$ is the root of the mean square current. Our results confirm that the exchange of energy is local, with larger values of $D_{i}$ localized in very small volume filling structures. This evidence and the presence of non-Gaussian PDFs of the dissipation proxies suggest that magnetic reconnection produces small-scale current sheets which are the site of strong events of energy exchange between fields and particles. Concisely stated, all these statistics indicate that dissipation in a reconnection event is intermittent. A similar conclusion was reached by Wan et al. (2012) who examined the electron frame dissipation surrogate conditioned on the magnitude of the current density.

Figures 8 and 9 show the statistics of dissipation proxies presented in figure 7 computed in sub-boxes located in the two reconnection outflows (see figure 6). NonGaussian statistics and the increasing conditioned average of the dissipation proxies indicate that intermittent turbulence is at play in both reconnection outflows.

\section{Energy exchanges in reconnection outflows}

As shown in figure 5, the region of the outflow is characterized by intense energy exchange $(\boldsymbol{J} \cdot \boldsymbol{E})$. Recently, the energy budget has been analysed in detail (Lapenta 

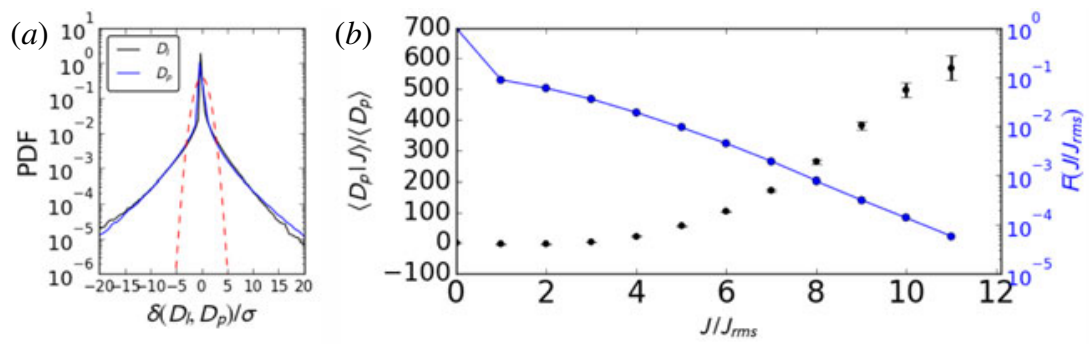

FIgURE 7. PDFs of $D_{l}$ and $D_{i}(a)$. Red dashed lines represent the normalized Gaussian curve. Mean $D_{i}$ conditioned on local current density thresholds and (right axis) fraction $F$ of the full box data used to compute the averages $(b)$.
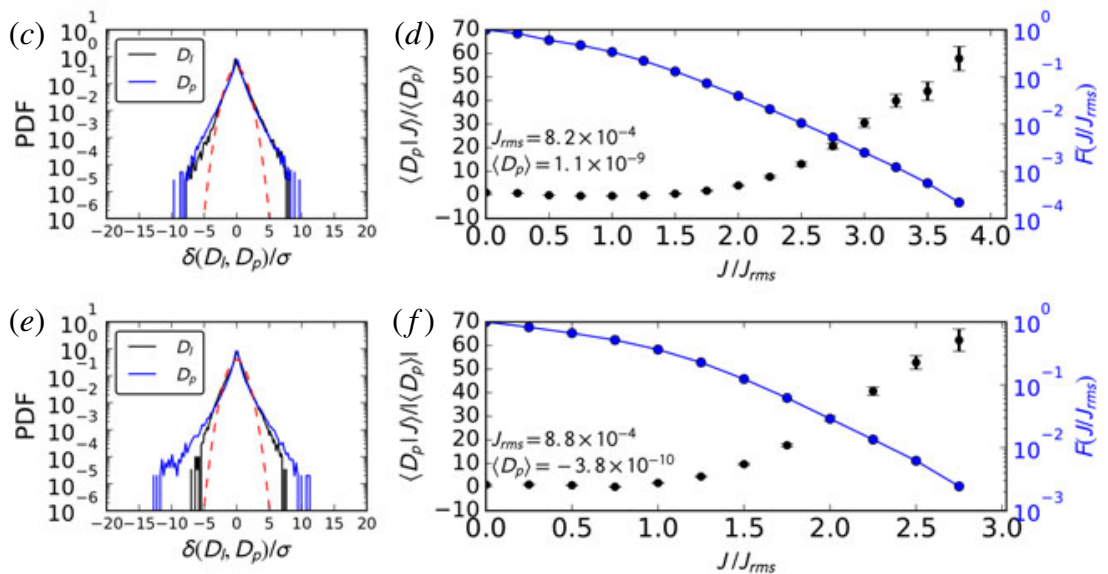

FIgURE 8. PDFs of $D_{l}$ and $D_{i}$ in $B O X_{1}(c), B O X_{2}(e)$. Conditioned average of $D_{i}$ and filling factors $F$ in $B O X_{1}(d), B O X_{2}(f)$ (left outflow).

et al. 2016b) and a large fraction of the energy is deposited as particle energization, while a significant fraction is also transported by the Poynting flux.

Figure 10 reports the ion temperature at the end of the run. Ions are generally not magnetized in the reconnection region and projecting the pressure tensor in the parallel and perpendicular direction relative to the magnetic field is not productive. Ion energization in reconnection outflows and in reconnection fronts have been analysed in theory and in simulation (Aunai, Belmont \& Smets 2011; Pan et al. 2012; Birn et al. 2013; Lapenta et al. 2016a). Complex processes are at play, requiring a full analysis of the phase space and of single particle trajectories to detect with accuracy the specific mechanisms accelerating the particles (Eastwood et al. 2015).

Figure 10 reports the three different kinetic temperatures obtained from the pressure tensor: $T_{j}=P_{j j} / \rho_{i}$, for $j=x, y, z$. The primary region of reconnection tends to heat the ions primarily in the $y$ direction. This effect is due to the mixing of the two populations of ions coming from above and below from the inflow towards the reconnection region. In the outflow, instead, the plasma outflowing along the $x$-direction mixes with the plasma in the medium causing apparent heating in the $x$-direction (Aunai et al. 2011). Heating in the $z$ direction is present both in the region of primary reconnection, where it is due to the acceleration of non-magnetized ions 

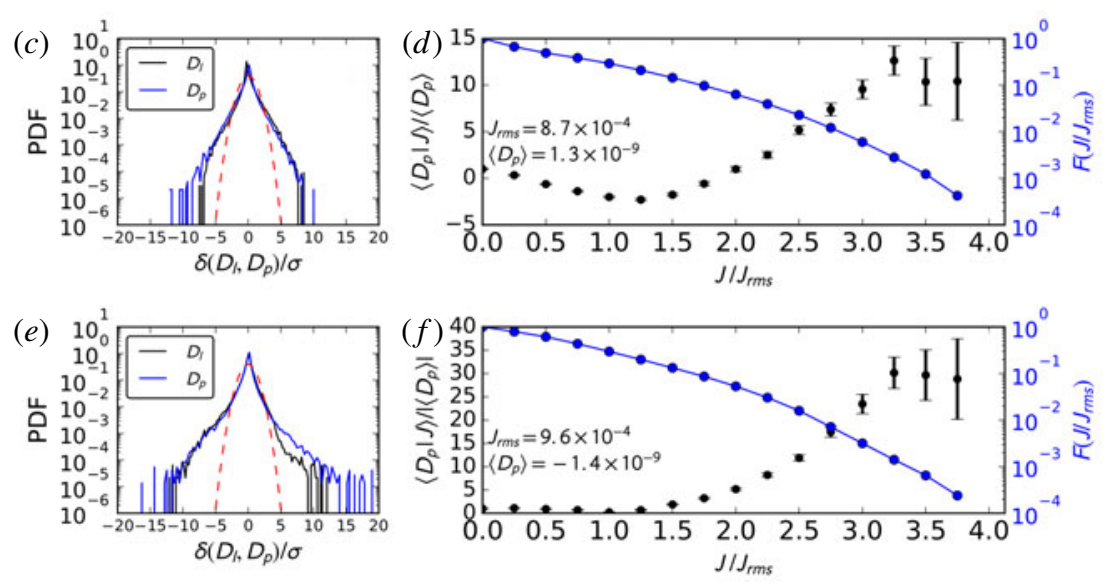

FIgURE 9. PDFs of $D_{l}$ and $D_{i}$ in $B O X_{3}(c), B O X_{4}(e)$. Conditioned average of $D_{i}$ and filling factors $F$ in $\mathrm{BOX}_{3}(d), \mathrm{BOX}_{4}(f)$ (right outflow).

in the reconnection region due to the reconnection electric field (Moses, Finn \& Ling 1993; Divin et al. 2010), and in the region of the outflows, where it is a consequence of the instabilities in the outflows. These effects however should not be interpreted as heating in the meaning of increasing thermodynamic temperature. The plasma is far from Maxwellian and what appears as heating in the kinetic temperature (i.e. the second-order moment of the distribution) is in reality the presence of multiple interpenetrating populations.

Figure 11 shows a volume rendering of the full 3-D velocity probability distribution for the ions. The distribution is anisotropic and contains multiple populations. When the second-order moment is taken to measure a kinetic temperature, the result can be misleading because multiple beams, each with its own temperature, appear as a single plasma with a combined temperature much higher than that of the beams. However, this is not a process of heating but one of bulk acceleration of ion populations. In a recent study, each ion component has been tracked back in time to its origin (Eastwood et al. 2015). Each component originates from different regions and their trajectories brought them to the same location but with different speeds.

Similarly, figure 12 show the parallel and perpendicular electron temperature. The electrons are mostly magnetized and it is more convenient to report the electron temperatures in magnetic coordinates rather than along geometrical axes. The region of primary reconnection causes parallel heating (Ricci, Lapenta \& Brackbill 2003). The cause is the reconnection electric field that accelerates the electrons along the $z$ direction (Wan et al. 2008; Divin et al. 2010): in this region the guide field is the only field present and the acceleration is parallel. The region of secondary instabilities in the outflow shows strong parallel and perpendicular energization caused by the conversion of electromagnetic energy (i.e. $\boldsymbol{J}_{e} \cdot \boldsymbol{E}$ ) (Lapenta et al. 2014a, 2016b).

The electron distribution is typically far smoother than the ion distribution due to the higher thermal speed. However, in the region of the secondary front instability, even the electron distribution becomes complex. Figure 13 shows a volume rendering of the full 3-D velocity probability distribution for the electrons computed as described above for the ions. On a large scale the distribution is bi-Maxwellian with different parallel and perpendicular temperatures. Within it we can observe multiple electron 
(a)
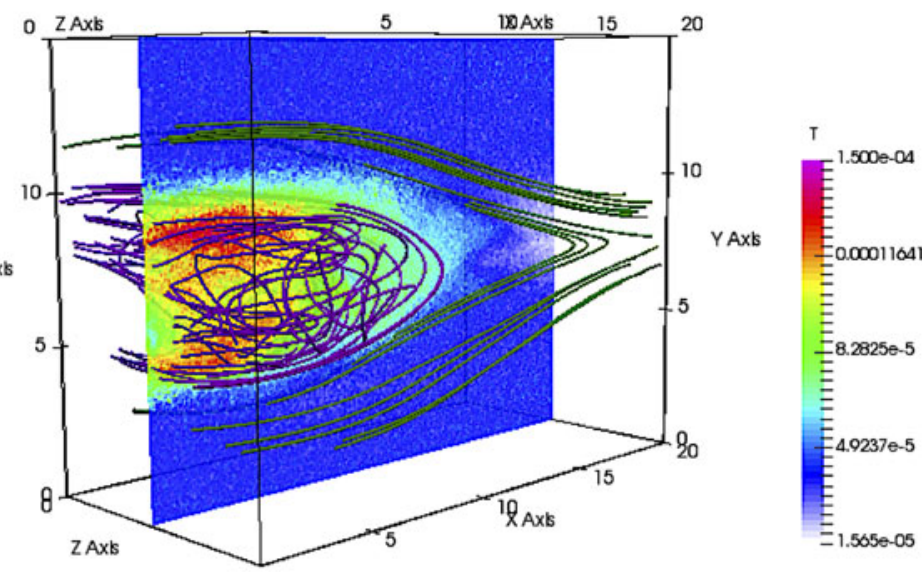

(b)

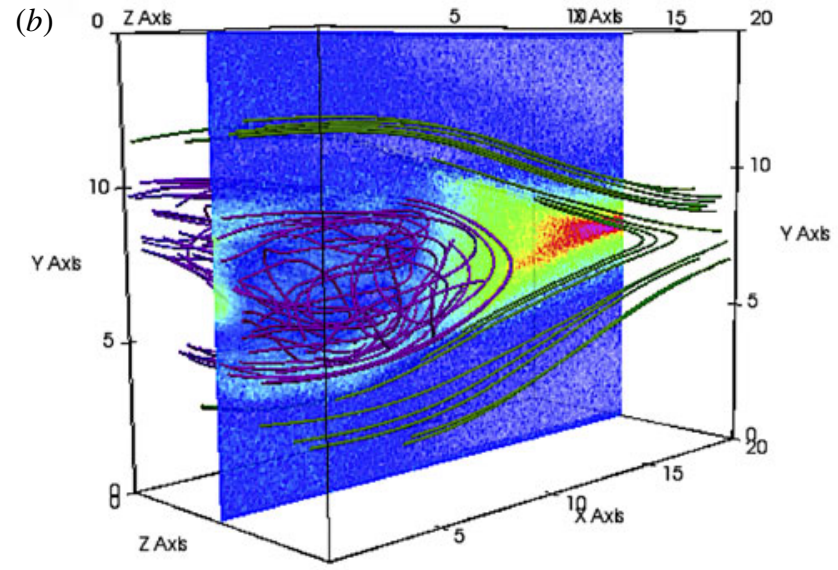

20

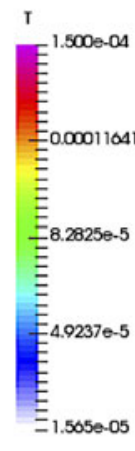

(c)
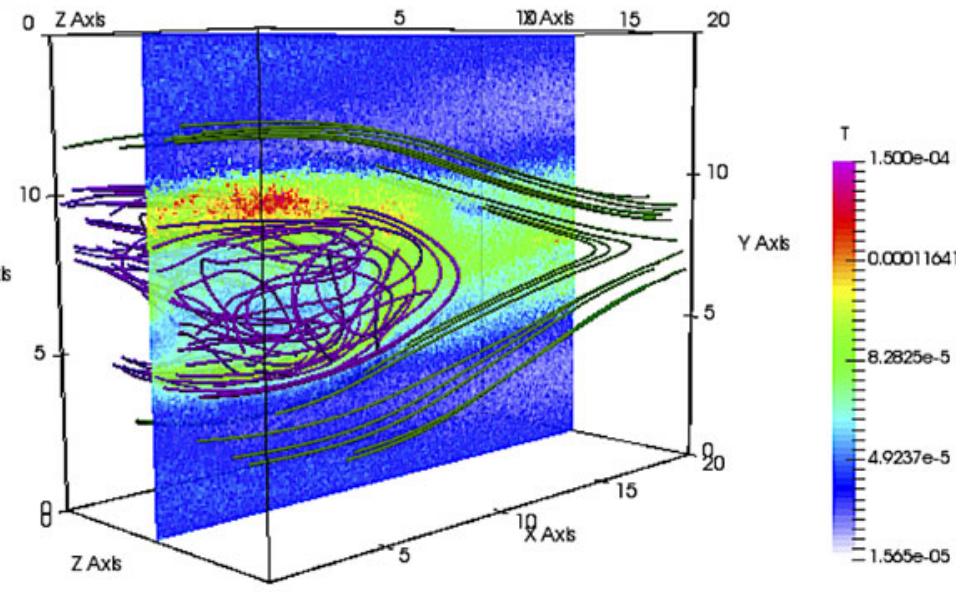

FIGURE 10. Ion temperature in the outflow: from top to bottom: $T_{x}(a), T_{y}(b)$ and $T_{z}(c)$. The left half of the domain is reported at the final time along with selected field lines. 


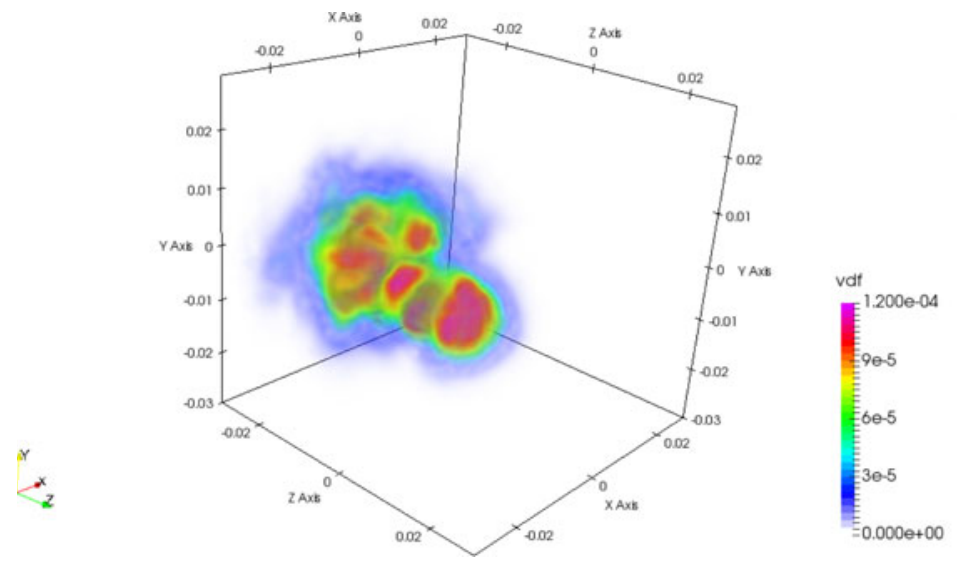

FIGURE 11. Volume rendering of the ion velocity probability distribution $f_{i}\left(v_{x}, v_{y}, v_{z}\right)$ at the position $x / d_{i}=10.32, y / d_{i}=7.5, z / d_{i}=5$, obtained by averaging over particles contained within a box centred at that location and with side $0.5 d_{i}$.

populations caused by electron acceleration by the electric field, directed primarily in the directions $z$ (reconnection electric field) and $y$ (Hall electric field) (Wan \& Lapenta 2008). Acceleration is not present in the $x$ direction where in fact there are no strong macroscopic electric fields.

The magnetic field lines show a chaotic behaviour: this condition makes it possible for particles moving along chaotic filed lines to access new acceleration regions of space (Dahlin, Drake \& Swisdak 2017), possibly encountering multiple reconnection sites and increasing their energy in steps.

\section{Conclusions and future directions}

The analysis of reconnection outflows in the present case of a weak guide field (1/10 of the main reconnecting field) show the development of an instability in the lower hybrid regime. In the present case, the instability has at least two components. The first, is due to the presence of density gradients formed in the pile-up region where the outflow meets the ambient plasma. The second is the pre-existing velocity shears due to the differential velocity between the Harris plasma and the ambient plasma (Lapenta, Brackbill \& Daughton 2003; Karimabadi et al. 2003; Ricci et al. 2004; Ricci, Lapenta \& Brackbill 2004). The first instability leads to a RayleighTaylor-type interchange instability in the lower hybrid range, while the latter leads to a kinking of the current layer.

Both instabilities feed the onset of a turbulent cascade with the presence of coherent structures and intermittency. The outflows becomes host to secondary reconnection sites where the magnetic field topology becomes chaotic (Lapenta et al. 2015).

We investigate here the effect of these processes on the energization of particles. The ions and the electrons are energized not only in the primary reconnection site but also, and in some cases predominantly, in the reconnection outflows. Particle energization can be linked to the electric fields operating on the particles. Electric fields do not heat particles in the statistical meaning of increasing their thermal spread, rather they coherently energize all particles, creating beams. Beams originating from different regions interact and interpenetrate creating distribution functions with multiple populations. 

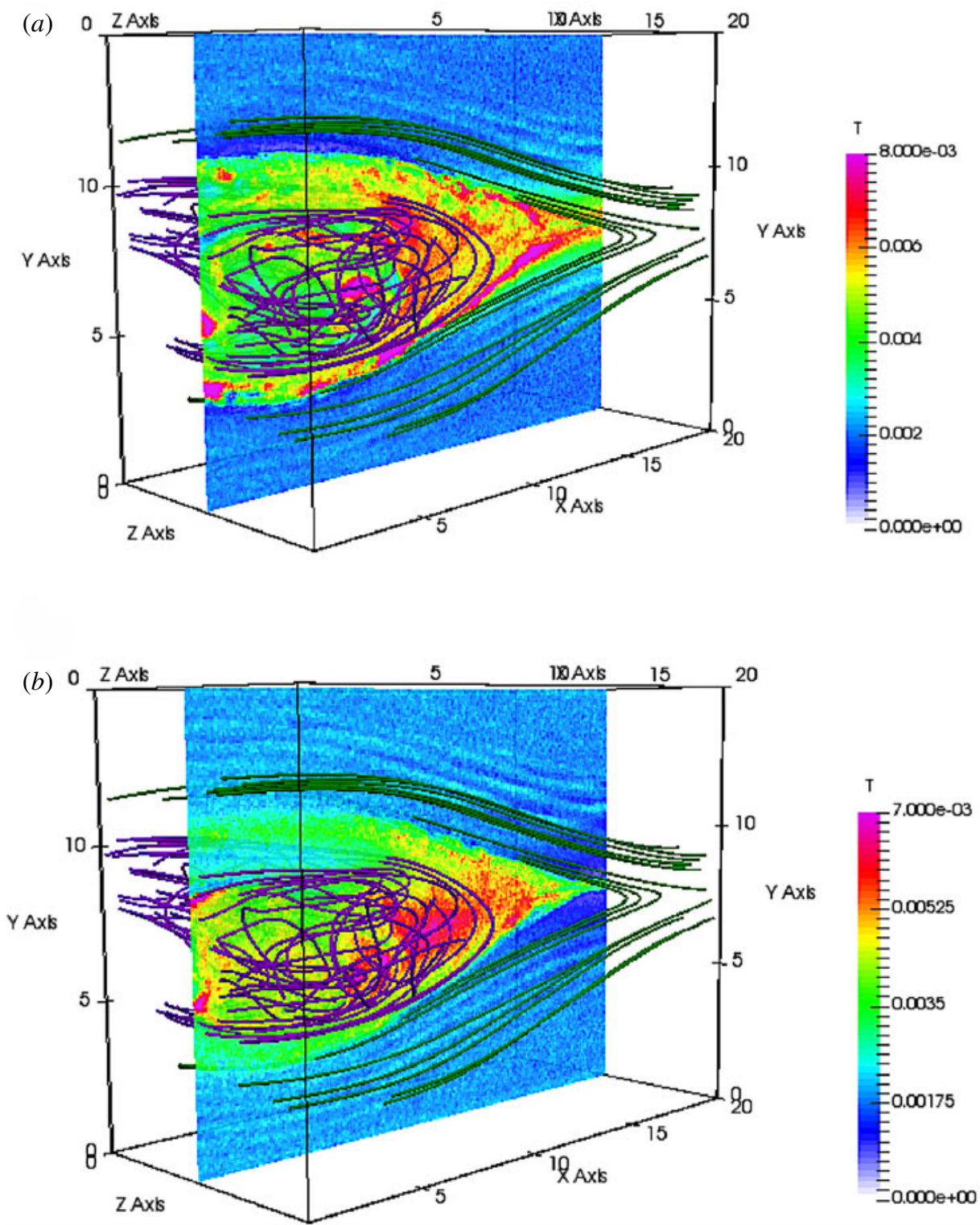

FIGURE 12. Electron parallel $(a)$ and perpendicular $(b)$ temperature in the outflow. The left half of the domain is reported at the final time along with selected field lines.

The end result is that the second-order moment of the distribution is increased but the process cannot be interpreted as heating proper but rather as the presence of very non-Maxwellian distributions with multiple beams.

\section{Acknowledgements}

The present work was supported by the Onderzoekfonds KU Leuven (Research Fund KU Leuven, GOA scheme and Space Weaves RUN project), by NASA's grant NNX08AO84G and by the US Air Force EOARD Award no. FA9550-14-1-0375. 


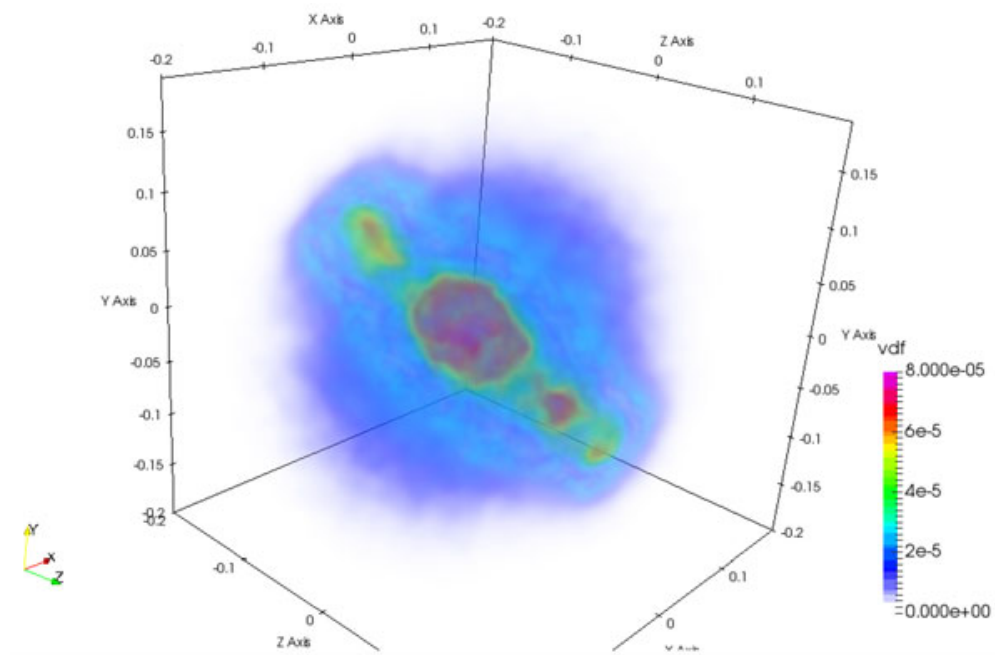

FIGURE 13. Volume rendering of the electron velocity probability distribution $f_{e}\left(v_{x}, v_{y}, v_{z}\right)$ at the position $x / d_{i}=10.32, y / d_{i}=7.5, z / d_{i}=5$, obtained by averaging over particles contained within a box centred at that location and with side $0.5 d_{i}$.

This research used resources of the National Energy Research Scientific Computing Center, which is supported by the Office of Science of the US Department of Energy under Contract no. DE-AC02-05CH11231. Additional computing has been provided by NASA NAS and NCCS High Performance Computing, by the Flemish Supercomputing Center (VSC) and by PRACE Tier-0 allocations.

\section{REFERENCES}

Aunai, N., Belmont, G. \& Smets, R. 2011 Proton acceleration in antiparallel collisionless magnetic reconnection: Kinetic mechanisms behind the fluid dynamics. J. Geophys. Res. 116 (A9).

Bhattacharjee, A., Huang, Y.-M., Yang, H. \& Rogers, B. 2009 Fast reconnection in highLundquist-number plasmas due to the plasmoid Instability. Phys. Plasmas 16 (11), 112102.

Birn, J., Drake, J. F., Shay, M. A., Rogers, B. N., Denton, R. E., Hesse, M., Kuznetsova, M., Ma, Z. W., Bhattacharjee, A., Отto, A. et al. 2001 Geospace environmental modeling (GEM) magnetic reconnection challenge. J. Geophys. Res. 106 (A3), 3715-3719.

Birn, J, Hesse, M, NAKAmura, R \& Zaharia, S 2013 Particle acceleration in dipolarization events. J. Geophys. Res. 118 (5), 1960-1971.

Biskamp, D. 2000 Magnetic Reconnection in Plasmas. Cambridge University Press.

Bulanov, S. V., SAKAI, J. \& SYROVATSKII, S. I. 1979 Tearing-mode instability in approximately steady MHD configurations. Fiz. Plazmy 5, 280-290.

Burch, J. L., Torbert, R. B., Phan, T. D., Chen, L.-J., Moore, T. E., Ergun, R. E., Eastwood, J. P., Gershman, D. J., CAssak, P. A., Argall, M. R. et al. 2016 Electronscale measurements of magnetic reconnection in space. Science 352 (6290), aaf2939.

Dahlin, J. T., Drake, J. F. \& Swisdak, M. 2017 The role of three-dimensional transport in driving enhanced electron acceleration during magnetic reconnection. Phys. Plasmas 24 (9), 092110; AIP Publishing.

Daughton, W., Roytershteyn, V., Karimabadi, H., Yin, L., Albright, B. J., Bergen, B. \& BowERS, K. J. 2011 Role of electron physics in the development of turbulent magnetic reconnection in collisionless plasmas. Nat. Phys. 7 (7), 539-542. 
Divin, A., Khotyaintsev, Y. V., Vaivads, A. \& André, M. $2015 a$ Lower hybrid drift instability at a dipolarization front. J. Geophys. Res 120 (2), 1124-1132.

Divin, A., Khotyaintsev, Y. V., Vaivads, A., André, M., Markidis, S. \& Lapenta, G. $2015 b$ Evolution of the lower hybrid drift instability at reconnection jet front. J. Geophys. Res. 120 (4), 2675-2690.

Divin, A., Markidis, S., Lapenta, G., Semenov, V. S., Erkaev, N. V. \& Biernat, H. K. 2010 Model of electron pressure anisotropy in the electron diffusion region of collisionless magnetic reconnection. Phys. Plasmas 17 (12), 122102.

Eastwood, J. P., Goldman, M. V., Hietala, H., Newman, D. L., Mistry, R. \& Lapenta, G. 2015 Ion reflection and acceleration near magnetotail dipolarization fronts associated with magnetic reconnection. J. Geophys. Res. 120 (1), 511-525.

Eastwood, J. P., Phan, T. D., Bale, S. D. \& Tuulin, A. 2009 Observations of turbulence generated by magnetic reconnection. Phys. Rev. Lett. 102 (3), 035001.

Fu, H. S., Vaivads, A., Khotyaintsev, Y. V., André, M., Cao, J. B., Olshevsky, V., EASTwOod, J. P. \& Retinò, A. 2017 Intermittent energy dissipation by turbulent reconnection. Geophys. Res. Lett. 44, 37-43.

Goldman, M. V., Newman, D. L. \& Lapenta, G. 2016 What can we learn about magnetotail reconnection from 2D PIC Harris-sheet simulations? Space Sci. Rev. 199 (1-4), 651-688.

Greene, J. M. 1992 Locating three-dimensional roots by a bisection method. J. Comput. Phys. 98, 194-198.

Guzdar, P. N., Hassam, A. B., Swisdak, M. \& Sitnov, M. I. 2010 A simple MHD model for the formation of multiple dipolarization fronts. Geophys. Res. Lett. 37 (20), L20102.

HARRIS, E. G. 1962 On a plasma sheath separating regions of oppositely directed magnetic field. Il Nuovo Cimento 23, 115-121.

Hesse, M. \& Schindler, K. 1988 A theoretical foundation of general magnetic reconnection. J. Geophys. Res. 93 (A6), 5559-5567.

Huang, Y.-M., Comisso, L. \& Bhattacharjee, A. 2017 Plasmoid instability in evolving current sheets and onset of fast reconnection. Astrophys. J. 849 (2), 75.

Karimabadi, H., Pritchett, P. L., Daughton, W. \& Krauss-Varban, D. 2003 Ion-ion kink instability in the magnetotail: 2. Three-dimensional full particle and hybrid simulations and comparison with observations. J. Geophys. Res. 108 (A11).

Kruskal, M. \& TUCK, J. L. 1958 The instability of a pinched fluid with a longitudinal magnetic field. Proc. R. Soc. Lond. A 245 (1241), 222-237.

Lapenta, G. 2008 Self-feeding turbulent magnetic reconnection on macroscopic scales. Phys. Rev. Lett. 100, 235001.

Lapenta, G., Ashour-Abdalla, M., Walker, R. J. \& El Alaoui, M. $2016 a$ A multiscale study of ion heating in earth's magnetotail. Geophys. Res. Lett. 43 (2), 515-524.

LAPENTA, G. \& BetTARINI, L. 2011 Self-consistent seeding of the interchange instability in dipolarization fronts. Geophys. Res. Lett. 38 (11).

Lapenta, G., BRACKBill, J. U.\& Daughton, W. S. 2003 The unexpected role of the lower hybrid drift instability in magnetic reconnection in three dimensions. Phys. Plasmas 10, 1577-1587.

Lapenta, G., Goldman, M., Newman, D., Markidis, S. \& Divin, A. 2014a Electromagnetic energy conversion in downstream fronts from three dimensional kinetic reconnectiona. Phys. Plasmas 21 (5), 055702.

Lapenta, G., Goldman, M. V., Newman, D. L. \& Markidis, S. $2016 b$ Energy exchanges in reconnection outflows. Plasma Phys. Control. Fusion 59 (1), 014019.

Lapenta, G., Markidis, S., Divin, A., Newman, D. \& Goldman, M. 2014b Separatrices: the crux of reconnection. J. Plasma Phys. 81 (1), 1-39.

Lapenta, G., Markidis, S., Goldman, M. V. \& Newman, D. L. 2015 Secondary reconnection sites in reconnection-generated flux ropes and reconnection fronts. Nat. Phys. 11 (8), 690-695.

LAPENTA, G., WANG, R. \& CAZzola, E. 2016c Reconnection separatrix: simulations and observations. In Magnetic Reconnection: Concepts and Applications (ed. W. D. Gonzalez \& E. N. Parker). pp. 315-344. Springer. 
Lottermoser, R.-F., Scholer, M. \& Matthews, A. P. 1998 Ion kinetic effects in magnetic reconnection: hybrid simulations. J. Geophys. Res. 103 (A3), 4547-4559.

Loureiro, N. F., Schekochinin, A. A. \& Cowley, S. C. 2007 Instability of current sheets and formation of plasmoid chains. Phys. Plasmas 14 (10), 100703.

MARKidis, S., LAPENTA, G. \& RizWAN-UdDin 2010 Multi-scale simulations of plasma with iPIC3D. Maths Comput. Simul. 80, 1509-1519.

Matteini, L., Alexandrova, O., Chen, C. H. K. \& Lacombe, C. 2017 Electric and magnetic spectra from MHD to electron scales in the magnetosheath. Mon. Not. R. Astron. Soc. 466, 945-951.

Moses, R. W., Finn, J. M. \& Ling, K. M. 1993 Plasma heating by collisionless magnetic reconnection: analysis and computation. J. Geophys. Res. 98 (A3), 4013-4040.

Nakamura, M. S., Matsumoto, H. \& Fujimoto, M. 2002 Interchange instability at the leading part of reconnection jets. Geophys. Res. Lett. 29 (8), 1247.

Olshevsky, V., Deca, J., Divin, A., Peng, I. B., Markidis, S., Innocenti, M. E., Cazzola, E. \& LAPENTA, G. 2016 Magnetic null points in kinetic simulations of space plasmas. Astrophys. J. 819 (1), 52.

Pan, Q., Ashour-Abdalla, M., El-Alaoui, M., Walker, R. J. \& Goldstein, M. L. 2012 Adiabatic acceleration of suprathermal electrons associated with dipolarization fronts. J. Geophys. Res. 117 (A12224).

Pucci, F., Servidio, S., Sorriso-Valvo, L., Olshevsky, V., Matthaeus, W. H., Malara, F., Goldman, M. V., Newman, D. L. \& Lapenta, G. 2017 Properties of turbulence in the reconnection exhaust: numerical simulations compared with observations. Astrophys. J. 841 (1), 60.

PuCCI, F. \& Velli, M. 2013 Reconnection of quasi-singular current sheets: the 'ideal' tearing mode. Astrophys. J. Lett. 780 (2), L19.

Ricci, P., Brackbill, J. U., Daughton, W. \& Lapenta, G. 2004 Influence of the lower hybrid drift instability on the onset of magnetic reconnection. Phys. Plasmas 11 (9), 4489-4500.

RicCI, P., LAPENTA, G. \& BRACKBILL, J. U. 2003 Electron acceleration and heating in collisioneless magnetic reconnection. Phys. Plasmas 10 (9), 3554-3560.

Ricci, P., LApenta, G. \& BrackBill, J. U. 2004 Structure of the magnetotail current: kinetic simulation and comparison with satellite observations. Geophys. Res. Lett. 31, L06801; doi:10.1029/2003GL019207.

ShafranOV, V. D. 1957 On equilibrium magnetohydrodynamic configurations. Zh. Eksp. Teor. Fiz. 33 (3), 710-722.

Skender, M. \& LAPENTA, G. 2010 On the instability of a quasiequilibrium current sheet and the onset of impulsive bursty reconnection. Phys. Plasmas 17, 022905.

Tenerani, A., Velli, M., Pucci, F., Landi, S. \& Rappazzo, A. F. 2016 'Ideally' unstable current sheets and the triggering of fast magnetic reconnection. J. Plasma Phys. 82 (5).

Vapirev, A. E., Lapenta, G., Divin, A., Markidis, S., Henri, P., Goldman, M. \& Newman, D. 2013 Formation of a transient front structure near reconnection point in 3-D PIC simulations. J. Geophys. Res. 118 (4), 1435-1449.

Wan, W. \& LApenta, G. 2008 Evolutions of non-steady-state magnetic reconnection. Phys. Plasmas 15 (10), 102302.

Wan, W., Lapenta, G., Delzanno, G. L. \& Egedal, J. 2008 Electron acceleration during guide field magnetic reconnection. Phys. Plasmas 15 (3), 032903.

Wan, M., Matthaeus, W. H., Karimabadi, H., Roytershteyn, V., Shay, M., Wu, P., Daughton, W., Loring, B. \& Chapman, S. C. 2012 Intermittent dissipation at kinetic scales in collisionless plasma turbulence. Phys. Rev. Lett. 109 (19), 195001.

Zenitani, S., Hesse, M., Klimas, A. \& Kuznetsova, M. 2011 New measure of the dissipation region in collisionless magnetic reconnection. Phys. Rev. Lett. 106 (19), 195003. 